\title{
STRATEGIES DE REPRODUCTION DU POISSON-CHAT Synodontis schall (BLOCH SCHNEIDER, 1801) (Siluriformes, Mochokidae) EN MILIEUX LACUSTRE ET FLUVIATILE (bassins Bia et Agnébi, Côte d'Ivoire)
}

\author{
K. C.BOUSSOU, M. OUATTARA, A. OUATTARA, D. DIOMANDE, Y. M. DIETOA et G. GOURENE \\ Université d'abobo-Adjamé, 02 BP801 Abidjan 02, Côte d'Ivoire \\ E-Mail : ouattara_bognan@hotmail.com
}

\begin{abstract}
RESUME
La reproduction du poisson-chat Synodontis schall a été étudiée en milieux lacustre et fluviatile, respectivement dans les rivières Bia et Agnébi. Le caractère permanent de l'eau, dans le lac a favorisé une plus grande période de reproduction de l'espèce dans la retenue, contrairement aux zones fluviatiles sujettes à des tarissements réguliers. Synodontis schall se reproduit dans le lac, d'avril à juin, puis d'octobre à novembre. Dans la rivière Agnébi et les parties fluviatiles de la Bia, l'espèce se reproduit seulement pendant un à deux mois (avril à mai). La taille de première reproduction du poisson, le taux de maturation des gonades et la fécondité ont été significativement plus élevés dans le lac qu'en milieux lotiques. Une analyse canonique (CANOCO), tenant compte à la fois des paramètres de la reproduction du poisson ainsi que des facteurs physico-chimiques des milieux étudiés, confirme que la reproduction et la croissance de l'espèce ont été nettement meilleures en milieu lacustre que dans les cours d'eau.
\end{abstract}

Mots clés : Reproduction, Synodontis schall, lac, rivière, Côte d'Ivoire.

\author{
ABSTRACT \\ REPRODUCTION STRATEGIES OF Synodontis schall (BLOCH SCHNEIDER, 1801) IN LAKE \\ AND RUNNING WATER ENVIRONMENTS (Bia and Agnébi bassins, côte d'IVOire)
}

A catfish breeding study (Synodontis schall) has been carried in lacustrin and fluviatile environments in Bia and Agnébi rivers, respectively. Water permanency in the lake favored a longer breeding season of this fish in the dam than in the river parts, where drought occurs frequently. Spawning season of the fish occured from april to june and from october to november in the lake. In the other study areas, the spawning ran just in one or two months (april to may). First maturity size, gonad maturity rate and fecundity are significantly higher in the lake than in the other water bodies studied. A canonical analysis (CANOCO), which takes into account both reproductive and environmental parameters, shown that the reproduction and the growth of Synodontis schall was better in the lake than in the running streams.

Key words : Reproduction, Synodontis schall, lake, river, Ivory Coast.

\section{INTRODUCTION}

La reproduction consiste en un transfert d'informations de la génération parentale à la descendance (Munro, 1990). Pour Wootton (1990), le succès de la reproduction dépend de l'endroit, du moment et des ressources utilisées. Concernant les poissons, Omanney (1964) a souligné qu'à l'heure venue, femelles et mâles respectivement pondent ovules et produisent du sperme dans l'eau où la fécondation a lieu. II est évident que dans de telles conditions, les pressions anthropiques de plus en plus fortes sur les milieux aquatiques, résultant le plus souvent de travaux d'aménagement, de surexploitation due à la pêche, peuvent mettre en danger la reproduction et les populations de poissons. 
Cependant, face à ces différents périls, un poisson peut développer des tactiques qui sont en réalité des variations par rapport au schéma de reproduction type de l'espèce, de manière à répondre, avec succès, aux modifications des facteurs environnementaux. II s'agit dans ce cas d'un comportement adaptatif à des conditions écologiques particulières, visant à assurer la survie de l'espèce (Lévêque, 1999 a). Le lieu où vit une espèce, c'est-à-dire son environnement physique, chimique et biologique est, pour cette espèce, le résultat d'un compromis entre différentes contraintes qui, pour une bonne part, sont liées à l'héritage des traits vitaux sélectionnés par la nature (Lévêque, 1999 b). Ces tactiques qui associent différents traits comprenant, notamment, la taille de première maturité sexuelle, la fécondité, le diamètre de l'ovule à la ponte, participent de ce que plusieurs auteurs appellent la stratégie de reproduction (Benech et Quensière, 1985).

Synodontis schall est un poisson benthique très apprécié pour la qualité de sa chair. Très peu d'études cependant ont été consacrées à la biologie de sa reproduction. La présente étude vise à établir les relations entre divers aspects de la reproduction du poisson et les conditions environnementales prévalant en milieux lentique et lotique.

\section{MATERIEL ET METHODES}

\section{MILIEUD'ETUDE}

L'échantillonnage des poissons a été réalisé dans les rivières Bia et Agnébi, toutes deux soumises à un régime équatorial de transition. La Bia prend sa source au Ghana, longe l'Est de la Côte d'Ivoire et se jette au Sud-Est dans la lagune Aby. Elle draine un bassin versant d'environ $9300 \mathrm{Km}^{2}$, avec un débit moyen à l'embouchure de $83 \mathrm{~m}^{3} \mathrm{~s}^{-1}$. Sur le cours de cette rivière a été construit en 1959, le premier barrage hydroélectrique du pays (Ayamé l) dont la retenue s'étend sur une superficie de $90 \mathrm{~km}^{2}$. Un second barrage (Ayamé II) a été construit en 1965 à $4 \mathrm{Km}$ en aval du premier avec un plan d'eau d'environ $7 \mathrm{Km}^{2}$ (Van den Bossche et Bernacsek, 1990). L'Agnébi est un cours d'eau sans barrage sur son lit principal. Cette rivière prend sa source à Bongouanou (Centre-Est du pays) et se jette dans la lagune Ebrié au niveau de la ville de Dabou. Son bassin versant couvre une superficie de $8700 \mathrm{~km}^{2}$.Son débit moyen à l'embouchure est de $50 \mathrm{~m}^{3} \mathrm{~s}^{-1}$.

\section{PARAMETRES PHYSICO-CHIMIQUES MESURES}

Les différents paramètres étudiés dans les deux rivières sont la température, l'oxygène dissous (mesurés à l'aide d'un oxymètre),le pH, le Taux de Solides Dissous (TDS), la conductivité électrique (évalués à l'aide d'un conductimètre), la transparence de l'eau mesurée à l'aide d'un disque de Secchi), la dureté totale, les teneurs en nitrites, nitrates, phosphore, calcium et ammonium (évalués à l'aide d'un spectrophotomètre).

\section{ECHANTILLONNAGE ICHTYOLOGIQUE ET ANALYSE DES CAPTURES}

L'échantillonnage mensuel des spécimens de Synodontis schall a porté sur deux cycles annuels, soit 24 mois. Sept stations ont été explorées dont quatre dans la Bia (Bianouan en amont, Bakro et Ayamé dans le lac, et Aboisso en aval), et trois dans l'Agnébi (Gbessé dans le cours supérieur, Pont-autoroute dans le cours moyen et Armébé dans le cours inférieur) (Figure 1). Au niveau de chaque station, les filets sont posés à 17 heures, visités le lendemain à 7 heures (pêche de nuit) et relevés à 13 heures (pêche de jour). Deux batteries de filets maillants de différentes mailles $(10 ; 12 ; 15 ; 20 ; 25$; $30 ; 35 \mathrm{~mm}$ ) ont été utilisées. Les effectifs étant trop faibles, à cause de la sécheresse, les résultats des trois stations de l'Agnébi ont été fusionnés. Chaque poisson a été identifié selon la clé de Paugy et Roberts (1992) et de Gourène et al., (1995). Les poissons ont été mesurés (longueur standard), puis pesés. La détermination de la taille de première reproduction (longueur standard à laquelle $50 \%$ des individus se reproduisent) a nécessité l'établissement de l'échelle de maturité sexuelle des gonades prélevées (Durand et Loubens, 1970). Pour Duponchelle et Légendre (2000), les individus qui ont atteint au moins le stade 3 de l'échelle de maturation sont considérés comme sexuellement matures. Pour déterminer la période de reproduction, le rapport gonadosomatique (RGS) mensuel (poids des gonades x 100/poids corporel éviscéré) a été calculé, ainsi que les pourcentages mensuels des stades avancés de la maturation sexuelle. Ces deux paramètres ont ensuite été couplés pour une meilleure précision sur la période de 


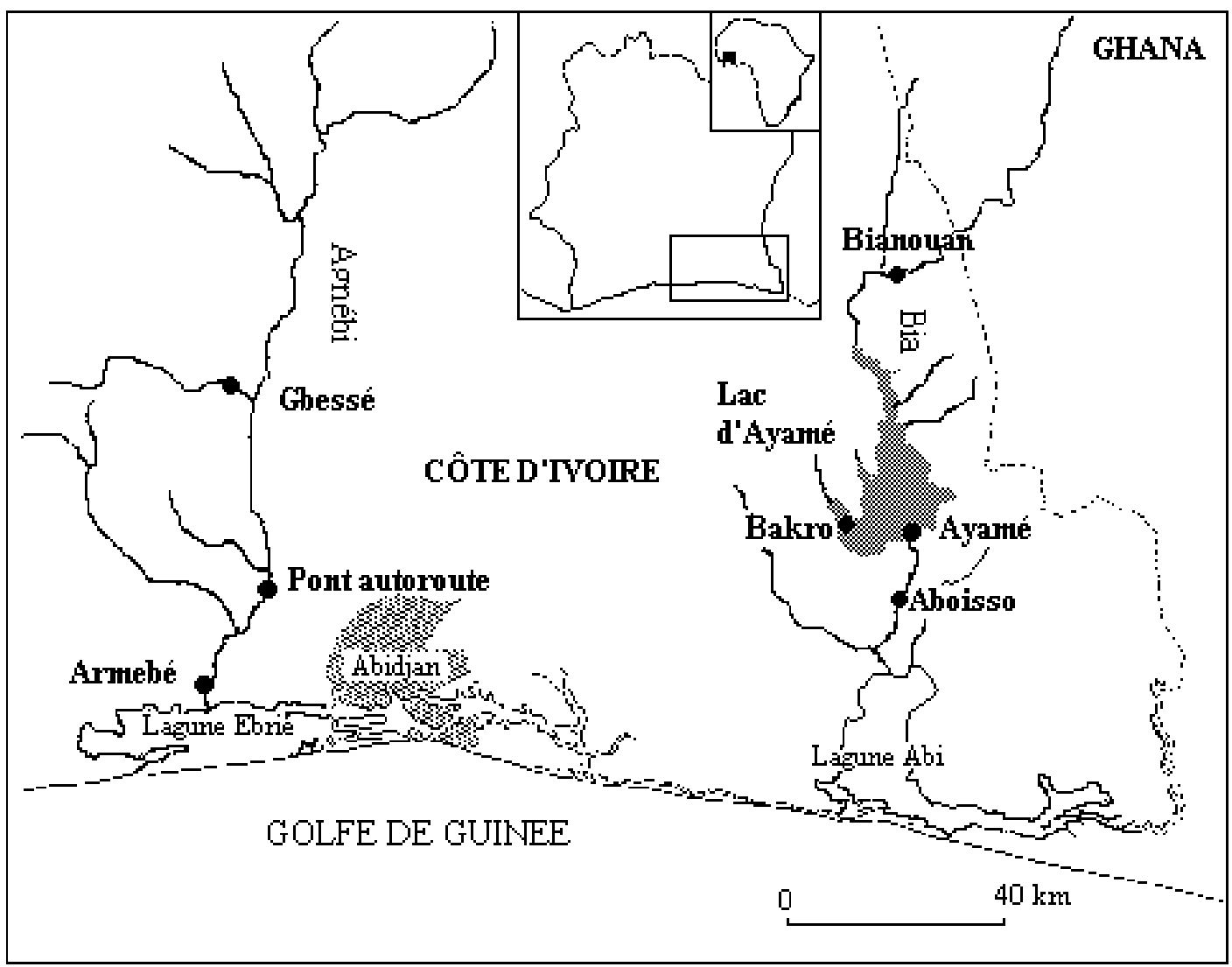

Figure 1 : Situation géographique des sites d'échantillonnage sur les rivières Bia et Agnébi

Geographical location of sampling sites in the Bia and Agnébi rivers (Gourène et al., 1999).

reproduction. Pour estimer le potentiel reproducteur et sa variabilité, la fécondité a été déterminée à partir des gonades de stade 4 récoltées dans les différentes stations. Une fraction précise $(0,2 \mathrm{~g})$ d'un ovaire mature a été prélevée et pesée puis les ovocytes comptés. Le nombre obtenu a été rapporté au poids total de la gonade ; ce qui traduit la fécondité estimée.

\section{ANALYSE STATISTIQUE}

Différents tests statistiques ont été utilisés : Des tests de variance pour comparer les moyennes des poids et de la fécondité des poissons prélevés dans les différentes stations; un test de médiane a été utilisé pour comparer les tailles des poissons. Enfin, une analyse canonique (CANOCO) a été appliquée aux paramètres de reproduction et les variables physico-chimiques enregistrés aux diverses stations d'échantillonnage en vue d'établir des corrélations entre ces facteurs écologiques en relation avec le milieu. La relation poids-longueur a été établie à l'aide de la formule $P=a L^{b}$ (Le Cren, 1951), où $P$ désigne le poids corporel du poisson, $L$ la longueur standard, a une constante et $b$, le coefficient d'allométrie. Après linéarisation de la formule : ( Log $P=\operatorname{Loga}+\operatorname{bLog} L)$, les valeurs de a et de $\mathrm{b}$ ont été déterminées par la méthode des moindres carrés. Cette relation a été établie année par année et par sexe. Chaque coefficient b a été comparé à la valeur 3 , qui traduit l'isométrie par le test de Student. Lorsque $b<3$, le poisson croît plus en longueur qu'en poids; lorsque $b>3$, le poisson croît plus en poids qu'en longueur et lorsque $b=3$, la croissance est isométrique. 


\section{RESULTATS}

\section{CARACTERISTIQUES PHYSICO-CHIMIQUES DES MILIEUX}

Les coefficients de corrélation entre les différentes variables physico-chimiques relevées dans les différents faciès sont donnés dans le tableau 1. Dans le lac (Tableau 1a), il apparaît une forte corrélation positive $(r>0,90)$ entre la température et la transparence, le $\mathrm{pH}$ et l'oxygène dissous et entre la conductivité et le Taux de Solides Dissous (TDS), les phosphates, la dureté totale et les ions calcium. II en est de même entre le TDS et les ions phosphates, les ions calcium et la dureté totale, entre les phosphates et les éléments azotés tels que les nitrates et les nitrites. Dans la rivière Agnébi (Tableau 1b) par ailleurs, on a observé une forte corrélation positive $(r>0,90)$ entre la conductivité, le TDS et la dureté totale, ainsi que entre les nitrates et la dureté totale.

Tableau 1a : Tests de corrélation entre les paramètres physico-chimiques relevés dans le lac d'Ayamé (basse Côte d'Ivoire).

Correlation test between standard physico-chemical parameters of Ayamé lake.

\begin{tabular}{|c|c|c|c|c|c|c|c|c|c|c|c|c|}
\hline \multirow{2}{*}{$\begin{array}{l}\text { Paramètres physico- } \\
\text { chimiques du lac d'Ayamé }\end{array}$} & \multicolumn{12}{|c|}{ Paramètres physico-chimiques du lac d'Ayamé } \\
\hline & $p H$ & Température $\left({ }^{\circ} \mathrm{C}\right)$ & $\mathrm{O}_{2}$ & Transparence & Conductivité & $\mathrm{PO}_{4}{ }^{3-}$ & $\mathrm{NH}_{4}^{+}$ & $\mathrm{NO}_{2}^{-}$ & $\mathrm{NO}_{3}^{-}$ & $T D$ & $\mathrm{Ca}^{2+}$ & $T D S$ \\
\hline$p H$ & 1 & & & & & & & & & & & \\
\hline Température & 0,45 & 1 & & & & & & & & & & \\
\hline $\mathrm{O}_{2}$ & 0,93 & 0,09 & 1 & & & & & & & & & \\
\hline Transparence & 0,54 & 0,99 & 0,20 & 1 & & & & & & & & \\
\hline Conductivité & $-0,73$ & $-0,94$ & $-0,42$ & $-0,97$ & 1 & & & & & & & \\
\hline $\mathrm{PO}_{4}^{3-}$ & $-0,89$ & $-0,80$ & $-0,67$ & $-0,86$ & 0,96 & 1 & & & & & & \\
\hline $\mathrm{NH}_{4}^{+}$ & $-0,16$ & $-0,95$ & 0,21 & $-0,92$ & 0,79 & 0,59 & 1 & & & & & \\
\hline $\mathrm{NO}_{2}^{-}$ & 0,63 & $-0,41$ & 0,87 & $-0,31$ & 0,07 & $-0,22$ & 0,66 & 1 & & & & \\
\hline $\mathrm{NO}_{3}^{-}$ & $-0,98$ & $-0,61$ & $-0,85$ & $-0,69$ & 0,84 & 0,96 & 0,34 & $-0,48$ & 1 & & & \\
\hline$T D$ & $-0,81$ & $-0,89$ & $-0,54$ & $-0,93$ & 0,99 & 0,99 & 0,71 & $-0,05$ & 0,90 & 1 & & \\
\hline $\mathrm{Ca}^{2+}$ & $-0,46$ & $-1,00$ & $-0,11$ & $-1,00$ & 0,95 & 0,81 & 0,95 & 0,39 & 0,62 & 0,90 & 1 & \\
\hline TDS & $-0,73$ & $-0,94$ & $-0,43$ & $-0,97$ & 1,00 & 0,96 & 0,79 & 0,07 & 0,85 & 0,99 & 0,94 & 1 \\
\hline
\end{tabular}

TD : dureté totale, TDS : taux de solide dissous

Tableau 1b : Test de corrélation entre les paramètres physico-chimiques relevés dans la rivière Agnébi (basse Côte d'Ivoire).

Correlation test between standard physico-chemical parameters of Agnébi river.

\begin{tabular}{|c|c|c|c|c|c|c|c|c|c|c|c|c|}
\hline \multirow[b]{2}{*}{$\begin{array}{l}\text { Paramètres physico-chimiques } \\
\text { de la rivière Agnébi }\end{array}$} & \multicolumn{12}{|c|}{ Paramètres physico-chimiques de la rivière Agnébi } \\
\hline & $\mathrm{pH}$ & $\begin{array}{c}\text { Température } \\
\left({ }^{\circ} \mathrm{C}\right)\end{array}$ & $\mathrm{O}_{2}$ & Transparence & Conductivité & $\mathrm{PO}_{4}^{3-}$ & $\mathrm{NH}_{4}^{+}$ & $\mathrm{NO}_{2}^{-}$ & $\mathrm{NO}_{3}^{-}$ & $T D$ & $\mathrm{Ca}^{2+}$ & TDS \\
\hline $\mathrm{pH}$ & 1 & & & & & & & & & & & \\
\hline Température & 0,15 & 1 & & & & & & & & & & \\
\hline $\mathrm{O}_{2}$ & 0,76 & 0,02 & 1 & & & & & & & & & \\
\hline Transparence & 0,53 & 0,73 & 0,21 & 1 & & & & & & & & \\
\hline Conductivité & $-0,26$ & $-0,97$ & $-0,14$ & $-0,74$ & 1 & & & & & & & \\
\hline $\mathrm{PO}_{4}^{3-}$ & $-0,10$ & $-0,52$ & 0,10 & $-0,61$ & 0,66 & 1 & & & & & & \\
\hline $\mathrm{NH}_{4}^{+}$ & $-0,05$ & $-0,17$ & 0,31 & $-0,64$ & 0,10 & 0,36 & 1 & & & & & \\
\hline $\mathrm{NO}_{2}^{-}$ & 0,29 & 0,00 & $-0,07$ & $-0,14$ & $-0,11$ & $-0,14$ & 0,43 & 1 & & & & \\
\hline $\mathrm{NO}_{3}^{-}$ & 0,08 & $-0,75$ & 0,31 & $-0,32$ & 0,78 & 0,58 & $-0,15$ & $-0,53$ & 1 & & & \\
\hline$T D$ & $-0,09$ & $-0,87$ & 0,09 & $-0,56$ & 0,93 & 0,75 & $-0,01$ & $-0,35$ & 0,94 & 1 & & \\
\hline $\mathrm{Ca}^{2+}$ & $-0,08$ & $-0,74$ & 0,36 & $-0,83$ & 0,71 & 0,69 & 0,70 & 0,00 & 0,59 & 0,69 & 1 & \\
\hline TDS & $-0,27$ & $-0,96$ & $-0,15$ & $-0,75$ & 1,00 & 0,66 & 0,10 & $-0,11$ & 0,77 & 0,93 & 0,70 & 1 \\
\hline
\end{tabular}

TD : dureté totale, TDS : taux de solide dissous

\section{CYCLE SAISONNIERDE LAREPRODUCTION}

La figure 2 montre l'évolution des variations de la maturation des gonades femelles (stades 3 et 4) par rapport au ratio gonado-somatique et de la pluviométrie. Au cours de la première année, des femelles matures ont été observées en amont de la rivière Bia (Figure 2a) en novembre, mars, avril, mai et juillet. En avril, ces femelles ont constitué l'essentiel de la population (100\%). Au cours des autres mois, leur proportion a été relativement faible $(<40 \%)$. Les valeurs du RGS moyen au cours de ces mêmes mois (avec une faible proportion 
de femelles matures) ont été inférieurs à $4,25 \%$. D'août à octobre, d'une part, de décembre à février et en juin, d'autre part, aucune femelle mature n'a été capturée. En seconde année, les femelles en maturation avancée ont été enregistrées en août et dans la période de mars à juillet. Les pourcentages les plus élevés sont apparus en août (100\%), en mai (100 \%) puis en juillet (66,6 \%). La valeur de l'index gonadique a été pratiquement nulle de septembre à février. Elle a augmenté régulièrement de mars à juillet et a marqué un pic en août (16,8\%).

Dans le milieu lacustre (Figure 2b), il y a eu une proportion élevée de femelles matures en aoûtseptembre, ensuite entre février et juin de la première année d'échantillonnage. Les fortes valeurs du RGS moyen, qui accompagnent ces états, ont été enregistrées en septembre $(12,2 \%)$, mars $(15,6 \%)$, avril $(12,5 \%)$ et en mai $(10 \%)$. En seconde année, les gonades matures ont été prélevées également en aoûtseptembre, puis sur la période allant de mars à juillet. Sur cette même période, les valeurs du
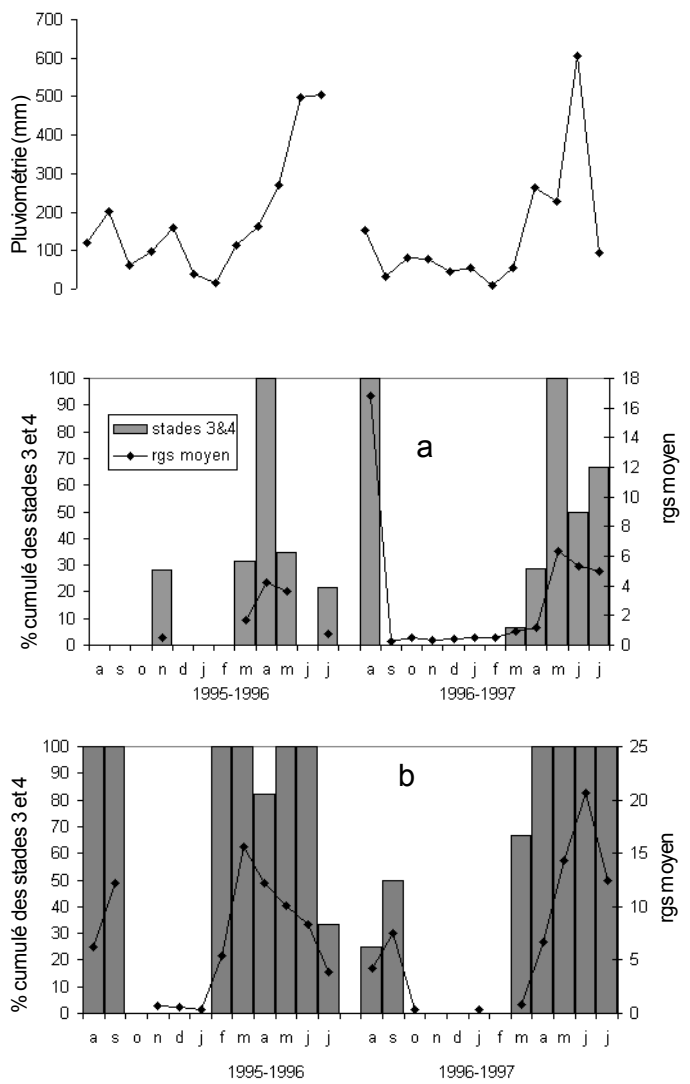

RGS moyen ont augmenté régulièrement pour atteindre un maximum en juin $(20,6 \%)$. La pluviométrie dans le bassin de la rivière Bia a présenté une période d'abondantes pluies qui s'est située globalement entre les mois d'avril et juillet. Les valeurs les plus basses des pluies ont été observées entre avril et juillet.

Au cours des deux années d'échantillonnage, aucun spécimen de Synodontis schall n'a été capturé en aval du lac.

Les assèchements fréquents de la rivière Agnébi (Figure 2c) n'ont pas permis un échantillonnage régulier. Au cours de la première année de pêche, les proportions les plus élevées des femelles matures ont été enregistrées en octobre et juillet, avec les valeurs respectives de 84 et $100 \%$. Les maxima de RGS moyen qui les accompagnent se sont élèvés respectivement à 12,35 et $4,23 \%$. Des observations ont été faites en novembre, mars et avril de l'année d'après, avec des valeurs respectives de 8,45 , 6,85 et $4,25 \%$ pour le RGS moyens et $65 \%$, $65 \%$ et $75 \%$ pour les femelles matures. En septembre, mars et juin de la première année, de même qu'en décembre et juin de la seconde année, aucune femelle mature n'a été pêchée.

Figure 2 : Evolution comparée du RGS (rapport gonado-somatique) et du \% des stades avancés de la maturité sexuelle des femelles de Synodontis schall dans les différents bassins étudiés au cours de deux années d'échantillonnage.

Comparative evolution of gonado-somatic index (GSI) and percentage of advanced stages of sexual maturity of Synodontis schall females at the different studied catchments areas during two years of sampling.
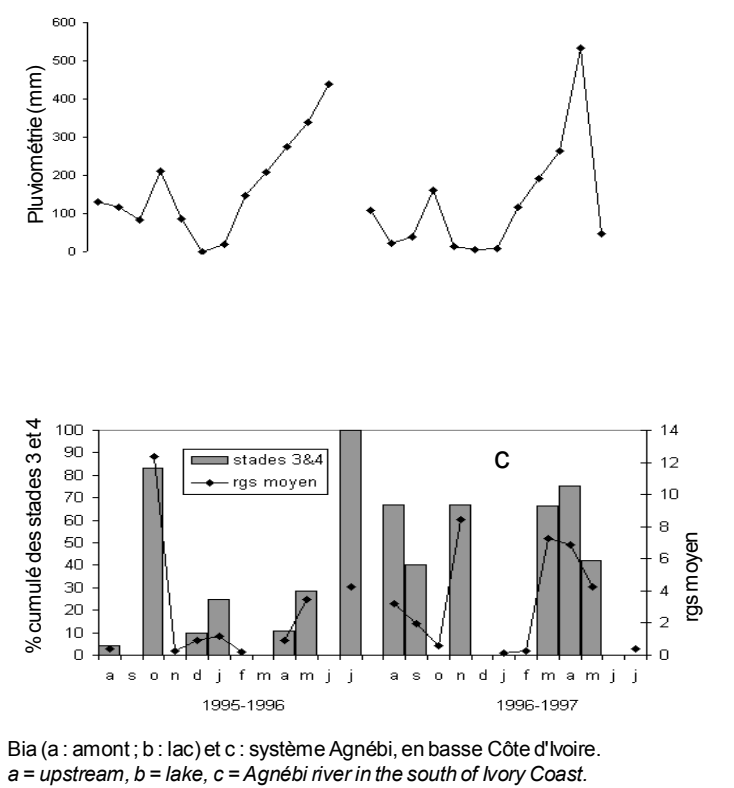


\section{TAILLE DE PREMIERE REPRODUCTION}

Concernant la L50 (Figure 3), elle n'a pu être déterminée qu'en deuxième année. Les valeurs obtenues sont de $184 \mathrm{~mm}$ dans le lac, $131 \mathrm{~mm}$ à l'amont et $145 \mathrm{~mm}$ dans la rivière Agnébi.

\section{FECONDITE CHEZ SYNODONTIS SCHALL}

Les moyennes de fécondité sont de 17232 $( \pm 10432)$ ovocytes en amont, et 20588 $( \pm 8956)$ ovocytes dans le lac et $9138( \pm 5587)$ ovocytes dans l'Agnébi. Le test de variance
(Tableau 2a) indique qu'il n'y a pas de différence significative entre l'amont et le lac. En revanche, la fécondité observée dans le lac est significativement plus élevée que celle enregistrée dans l'Agnébi. ( $p<0,05)$.

\section{ANALYSES STATISTIQUES}

\section{Evolution du poids des poissons capturés}

Les poids moyens des femelles prélevées dans le lac (121,5 g, année 1) et (154,14 g année 2) sont nettement supérieurs aux poids moyens
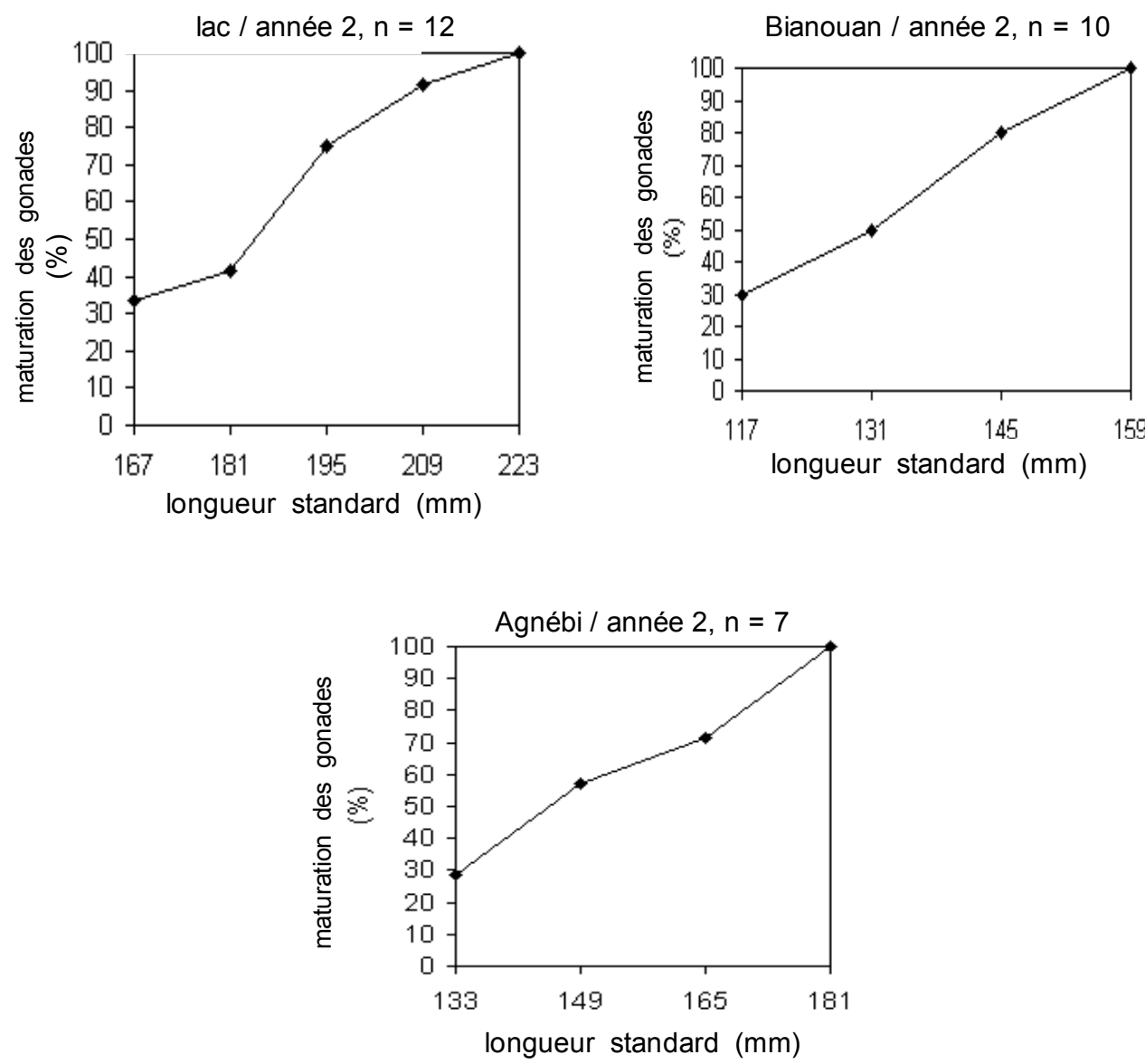

Figure 3 : Taille de première maturité sexuelle des femelles de Synodontis schall en milieux fluvial et lacustre dans les bassins côtiers de la Bia et de l'Agnébi (Côte d'Ivoire).

First sexual maturity size of S. schall females in river and lacustrin areas of the river basins of Bia and Agnébi (Côte d'lvoire). 
des femelles de la rivière Agnébi qui pèsent respectivement entre $85,88 \mathrm{~g}$ et $80,83 \mathrm{~g}$. Ils sont nettement plus élevés que ceux des femelles capturées en amont avec des valeurs moyennes de 46,27 et $47,92 \mathrm{~g}$ en première et deuxième année (Tableau 2b). Dans le même ordre et chez les mâles, les poids enregistrés dans le lac sont significativement plus élevés $(p<0,05)$ que ceux relevés dans les deux autres milieux, soit $87,47 \mathrm{~g}$ et $106,11 \mathrm{~g}$ dans le lac contre 38,9 $\mathrm{g}$ et $53 \mathrm{~g}$ en amont et 87,36 $\mathrm{g}$ et $71,86 \mathrm{~g}$ dans l'Agnébi. Entre l'amont de la Bia et l'Agnébi, la variation du poids corporel des poissons, tous sexes confondus, est irrégulière mais toujours inférieure au poids des poissons du lac.

Tableau 2a : Analyse de variance sur la fécondité des spécimens de Synodontis schall pêchés dans les différentes stations des systèmes fluvio-lacustres de la Bia et de l'Agnébi (Côte d'Ivoire), au cours de deux années de pêche.

Variance analysis on fecundity of fish S. schall caught at different stations of the the Bia and Agnébi rivers and lakes valley (Côte d'lvoire) during the two years of sampling.

\begin{tabular}{lccc}
\hline & Amont & Lac & Agnébi \\
\cline { 2 - 4 } & $\mathrm{M}=17232,67$ & $\mathrm{M}=20588,33$ & $\mathrm{M}=9138,777$ \\
\hline Amont & 1 & 1 & \\
lac & 0,79967535 & $\mathbf{0 , 0 1 6 4 9 9 6 4}$ & 1 \\
agnébi & 0,2937851 & & \\
\hline$(P<0,05 . \quad M=$ Moyenne $)$ & & \\
$(P<0,05 . M=$ mean $)$ &
\end{tabular}

Tableau 2b : Analyse de variance sur les poids corporels des spécimens de Synodontis schall pêchés dans les différentes stations des systèmes fluvio-lacustres de la Bia et de l'Agnébi (Côte d'Ivoire), au cours de deux années de pêche.

Variance analysis on fecundity (a) and body weight (b) of the fish, S. schall caught at different stations of the Bia and Agnébi rivers and lakes (Côte d'lvoire) during the two yearsof sampling.

\begin{tabular}{lccc}
\hline & \multicolumn{3}{c}{ Femelles année 1 } \\
\cline { 2 - 4 } & Amont & Lac & Agnébi \\
\cline { 2 - 4 } & $\mathrm{M}=46,272$ & $\mathrm{M}=121,50$ & $\mathrm{M}=85,883$ \\
\hline Amont & 1 & 1 & \\
lac & 0,000022 & 0,00022 & 1 \\
agnébi & 0,000034 & \multicolumn{3}{c}{ Femelles année 2 } \\
\hline \multicolumn{4}{c}{ Lac } \\
\hline Amóbi \\
\cline { 2 - 4 } & $\mathrm{M}=47,916$ & $\mathrm{M}=154,14$ & $\mathrm{M}=80,832$ \\
\hline lac & 1 & 1 & 1 \\
agnébi & 0,000113 & 0,000113 & \\
\hline
\end{tabular}

( $\mathrm{P}<0,05 . \quad M=$ Moyenne $)$.

( $P<0,05 . M=$ mean $)$.

\section{Evolution spatio-temporelle de la taille des poissons}

\begin{tabular}{|c|c|c|c|}
\hline & \multicolumn{3}{|c|}{ Mâles année 1} \\
\hline & Amont & Lac & Agnébi \\
\hline & $M=38,903$ & $M=87,486$ & $M=87,368$ \\
\hline Amont & 1 & & \\
\hline lac & 0,00813 & 1 & \\
\hline \multirow[t]{4}{*}{ agnébi } & 0,04146 & 0,99998 & 1 \\
\hline & \multicolumn{3}{|c|}{ Mâles année 2} \\
\hline & Amont & Lac & Agnébi \\
\hline & $M=53,004$ & $M=106,71$ & $M=71,868$ \\
\hline Amont & 1 & & \\
\hline lac & 0,000116 & 1 & \\
\hline agnébi & 0,16861845 & 0,00456 & 1 \\
\hline
\end{tabular}

Concernant les femelles, trois constats se dégagent : d'une année à l'autre, les tailles des femelles pêchées en amont ont été significativement ou très significativement inférieures à celles des femelles capturées dans le lac (Tableau 3). En effet, les tailles moyennes des femelles matures en amont ont varié entre $110 \mathrm{~mm}$ et $170 \mathrm{~mm}$, tandis que dans le lac,

les tailles extrêmes se situent entre $119 \mathrm{~mm}$ et $225 \mathrm{~mm}$;

Au cours des deux années d'échantillonnage, la taille des femelles pêchées en amont a été inférieure à celle des femelles provenant de la rivière Agnébi ( 125 contre $200 \mathrm{~mm}$ );

Entre les femelles capturées dans le lac et dans la rivière Agnébi, les variations des tailles n'ont pas été régulières d'une année à l'autre d'une part et d'un milieu à l'autre d'autre part. 
Tableau 3 : Test de médiane traduisant la variation de la taille des populations de Synodontis schall des rivières Bia et Agnébi (Basse Côte d'Ivoire) au cours de deux années.

Median test expressing standard length variations of Synodontis schall populations from the Bia and Agnébi rivers (south of Ivory Coast) during the two years of experiment.

\begin{tabular}{llllccc}
\hline & Désignation & médiane & $n$ & Chi-deux calculé & Chi-deux théorique & Significativité \\
\hline Année 1 & f1am-f1lac & 125 & $17 ; 40$ & 5,22 & 3,84 & $\mathrm{~S}^{*}$ \\
Femelles & f1lac-f1agn & 150 & $40 ; 25$ & 0,22 & 3,84 & $\mathrm{~ns}$ \\
& f1am-f1agn & 134 & $25 ; 17$ & 22,23 & 3,84 & $\mathrm{~S}^{* *}$ \\
\hline \multirow{3}{*}{ Mâles } & m1am-m1lac & 122 & $14 ; 19$ & 0,2 & 3,84 & $\mathrm{~ns}$ \\
& m1lac-m1agn & 150 & $19 ; 8$ & 0,51 & 3,84 & $\mathrm{~ns}$ \\
\hline Année 2 & m1am-m1agn & 123,5 & $14 ; 8$ & 5,46 & 3,84 & $\mathrm{~S}^{*}$ \\
Femelles & f2am-f2lac & 133 & $64 ; 28$ & 20,89 & 3,84 & $\mathrm{~S}^{* *}$ \\
& f2lac-f2agn & 157,5 & $28 ; 28$ & 14 & 3,84 & $\mathrm{~S}^{* *}$ \\
\hline \multirow{3}{*}{ Mâles } & f2am-f2agn & 130 & $64 ; 28$ & 4,11 & 3,84 & $\mathrm{~S}^{*}$ \\
& m2am-m2lac & 141 & $29 ; 17$ & 15,76 & 3,84 & $\mathrm{~S}^{* *}$ \\
& m2lac-m2agn & 152 & $17 ; 12$ & 7,45 & 3,84 & $\mathrm{~S}^{*}$ \\
\hline
\end{tabular}

$\mathrm{n}=$ effectif $;$ obs = observé $;$ théo = théorique $; \mathrm{s}=$ significatif $; \mathrm{ns}=$ non significatif $;$ ddl = degré de liberté $; \mathrm{f} 1=$ femelles année 1 ; f2 = femelles année $2 ;$ Bian = Bianouan $;$ agn = Agnébi.

$\mathrm{n}=$ total population $;$ obs = observed; th = theoric $; \mathrm{s}=$ significant $; \mathrm{ns}=$ non significant $; \mathrm{ddl}=$ liberty degree of freedom, $\mathrm{f} 1=$ females from first year $1 ; \mathrm{f} 2=$ females from second years $2 ;$ Bian $=$ Bianouan $;$ agn $=$ Agnébi .

\section{Analyse canonique}

Les résultats (Figure 4) montrent que la plus grande partie de la variabilité est donnée par l'axe I (62,6\%) et l'axe II (37,4\%). La transparence et la température placées dans la partie négative de l'axe I (lac), les teneurs en oxygène et en calcium situées dans la partie positive (amont de la rivière $\mathrm{Bia}$ ), sont les facteurs écologiques qui influencent cet axe. On note une forte corrélation positive entre transparence et température. Le taux de solides dissous (TDS), la conductivité (CND), la dureté totale (TD) et le phosphore, situés dans la partie positive, déterminent le plus l'axe II. La corrélation entre ces facteurs reste très forte.
Les paramètres physico-chimiques qui n'apparaissent pas sur le graphique, sont celles qui ne subissent presque aucune variation spatiotemporelle. Dans le lac, le RGS, le taux de maturation et le poids corporel sont les paramètres de la reproduction les plus fortement exprimés et positivement corrélés à la transparence et à la température. A Bianouan, la fécondité a été le seul paramètre biotique observé et influencée positivement par l'oxygène dissous. Au niveau du système fluviatile Agnébi, le sex-ratio a été le seul paramètre distinctif. La corrélation entre cette variable et les paramètres physico-chimiques (le taux de solides dissous, la conductivité, la dureté totale et le phosphore) a été très faible. 


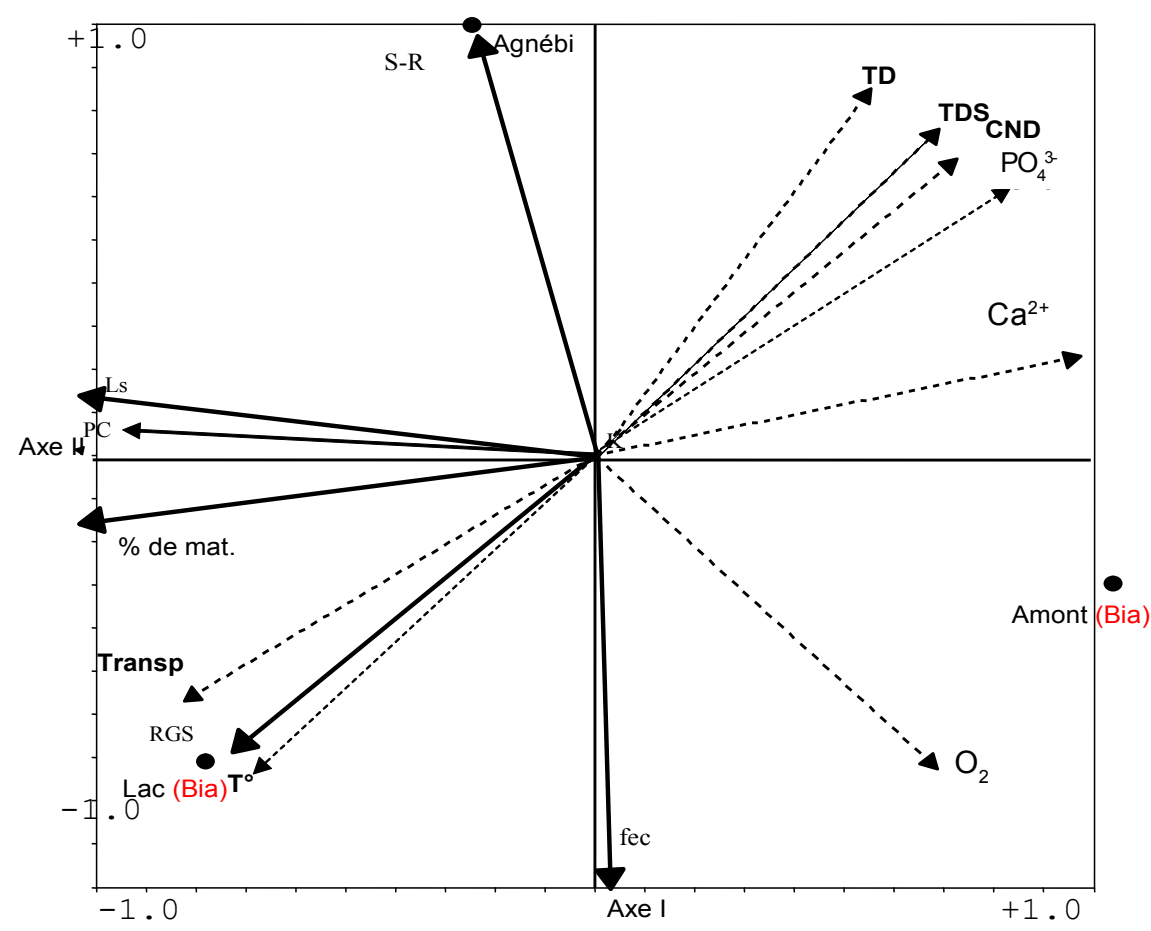

Figure 4 : Analyse canonique des correspondances (programme CANOCO) des paramètres biotiques de Synodontis schall et de 12 variables abiotiques dans les différentes stations des rivières Bia et Agnébi en basse Côte d'Ivoire au cours des deux années d'étude.

Canonical analysis of correspondance (RDA, program CANOCO) using reproduction parameters of Synodontis schall and 12 physical and chemical at the different stations of Bia and Agnébi rivers in the south of Ivory Coast during two years of experiment

Ls = longueur standard; Transp. = transparence, $\mathrm{CND}=$ conductivité, $\%$ de mat. = pourcentage de maturation; Fec = fécondité $; \mathrm{PC}=$ poids corporel $\mathrm{S}-\mathrm{R}=$ sex-ratio $; \mathrm{TD}=$ dureté totale $; \mathrm{CA} 2=$ calcium $; \mathrm{TDS}=$ taux de solides dissous $; \mathrm{O} 2$ = oxygène dissous $; \mathrm{PO} 43-=$ phosphates $; \mathrm{K}=$ coefficient de condition $;$ RDA $=$ Analyse de Redondance.

(Ls = standard length; Transp. = transparency, CND = conductivity).

\section{Relations poids-longueur des poissons}

Différentes relations poids-tailles ont été établies (Figure 5). Les différents coefficients b obtenus et leur niveau de signification (déterminé par le test de Student) sont consignés dans le tableau 4. Sur la base de ces éléments, il apparaît des variations spatio-temporelles du poids et de la taille des poissons. En première année d'échantillonnage, les femelles du lac et de
l'Agnébi croissent mieux en poids qu'en longueur (b > 3), contrairement à celles de l'amont qui elles, croissent mieux en longueur qu'en poids $(b<3)$. Cette observation est exactement applicable aux mâles de cette même année. En deuxième année, chez les femelles, aussi bien en amont dans le lac que dans l'Agnébi, il y a une meilleure croissance en longueur qu'en poids. Chez les mâles, seuls les spécimens capturés en amont présentent une meilleure croissance en poids qu'en longueur. 

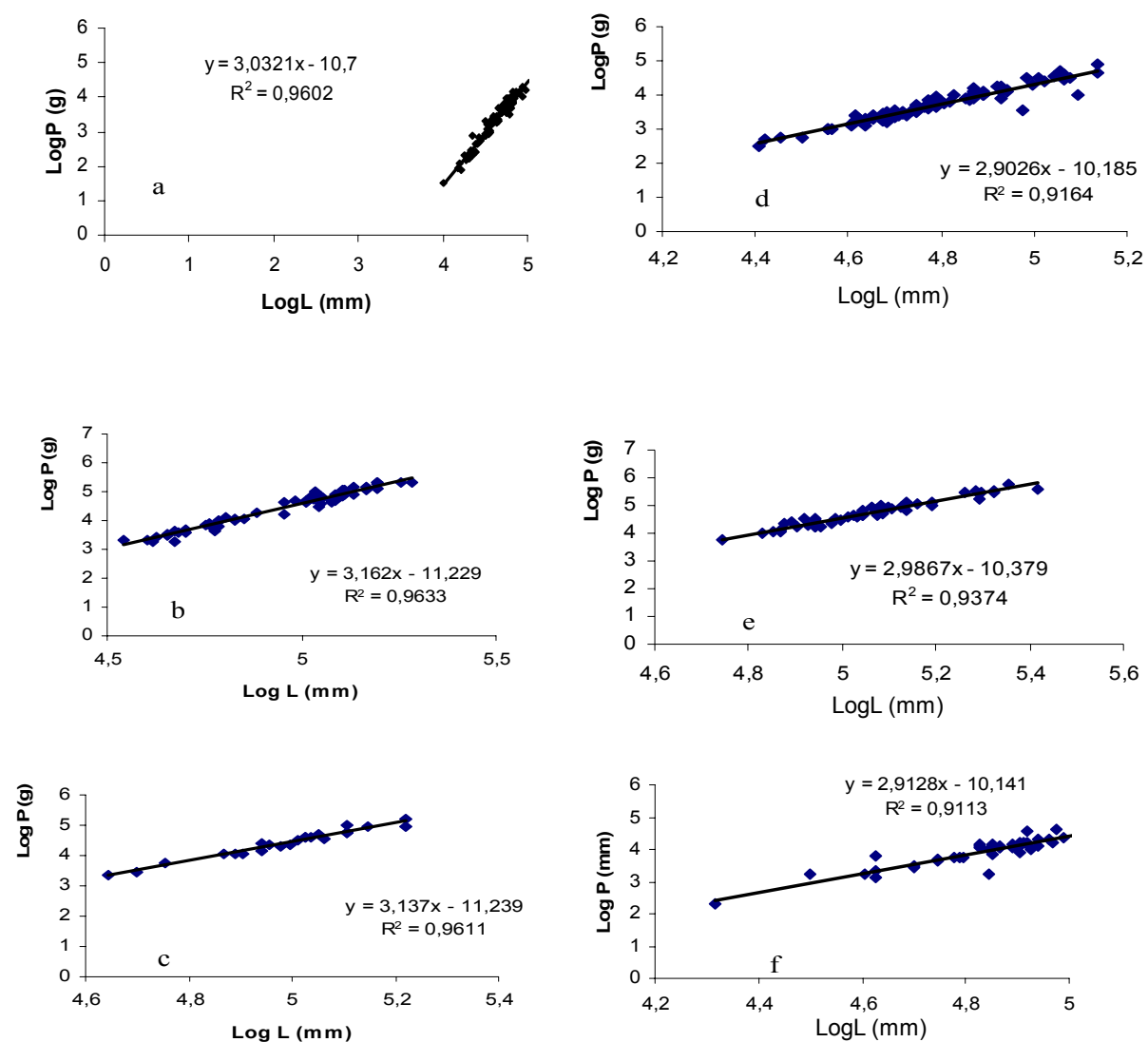

$\mathrm{a}=$ Bianouan $; \mathrm{b}=\mathrm{lac} ; \mathrm{c}=$ Agnébi $a=$ upstream $; b=$ lake $; c=$ Agnébi $P=$ poids $L=$ longueur standard

Figure 5 : Relations poids corporel- longueur standard de Synodontis schall en milieux fluvio-lacustres de la basse Côte d'Ivoire.

Relationships between body weight and standard length of Synodontis schall.

Tableau 4 : Résultat du test de Student exprimant les relations entre la longueur standard et le poids corporel des poissons Synodontis schall capturés dans les différentes stations des systèmes fluvio-lacustres de la Bia et de l'Agnébi (Côte d'Ivoire) au cours de deux années.

Results of Student test expressing the relationships between standard length and body weight parameters of fish caught at different stations of the Bia and Agnébi river lakes (Côte d'Ivoire) during the two years of sampling.

\begin{tabular}{|c|c|c|c|c|c|c|c|c|}
\hline & & Station & $\mathrm{n}$ & $r$ & $a$ & $\mathrm{~b}$ & $\mathrm{t}$ (calculé) & $\mathrm{t}(\mathrm{lu})$ \\
\hline \multirow{6}{*}{ 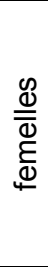 } & \multirow{3}{*}{ 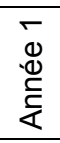 } & amont & 12 & 0,96 & 8,00E-05 & 2,76 & 10,84 & 2,718 \\
\hline & & lac & 27 & 0,97 & $1,12 \mathrm{E}-05$ & 3,2 & 19,95 & 2,779 \\
\hline & & Agnébi & 24 & 0,98 & 1,30E-05 & 3,14 & 23,10 & 2,807 \\
\hline & \multirow{3}{*}{ 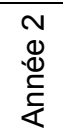 } & amont & 46 & 0,93 & 1,05E-05 & 2,69 & 16,78 & 2,576 \\
\hline & & lac & 22 & 0,97 & $3,20 \mathrm{E}-05$ & 2,97 & 17,84 & 2,819 \\
\hline & & Agnébi & 27 & 0,94 & 4,00E-05 & 2,87 & 14,17 & 2,779 \\
\hline \multirow{6}{*}{$\frac{\mathscr{\mathscr { D }}}{\frac{\mathbb{\sigma}}{\mathscr{C}}}$} & \multirow{3}{*}{ 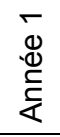 } & amont & 10 & 0,93 & $3,00 E-05$ & 2,95 & 6,89 & 2,262 \\
\hline & & lac & 15 & 0,98 & 1,05E-05 & 3,2 & 17,76 & 2,977 \\
\hline & & Agnébi & 6 & 0,98 & 8,00E-07 & 3,69 & 9,85 & 1,476 \\
\hline & \multirow{3}{*}{ 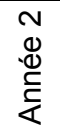 } & amont & 20 & 0,98 & 1,60E-05 & 3,08 & 20,89 & 2,861 \\
\hline & & lac & 15 & 0,90 & 1,50E-03 & 2,67 & 7,62 & 2,977 \\
\hline & & Agnébi & 15 & 0,94 & $7,00 \mathrm{E}-05$ & 2,76 & 9,93 & 2,977 \\
\hline
\end{tabular}

$\mathrm{n}=$ effectif $; \mathrm{r}=$ coefficient de corrélation ; $\mathrm{a}=$ pente de la droite de régression $; \mathrm{b}=$ coefficient d'allométrie ; $\mathrm{t}=\mathrm{t}$ de Student $;$ calc $=$ calculé. 


\section{DISCUSSION}

Les données sur la reproduction, obtenues sur la base des paramètres spécifiques, montrent plusieurs périodes d'activités sexuelles. Mais selon Moreau (1979), la période de reproduction est celle où la majorité des individus se reproduisent. Ainsi, Synodontis schall se reproduit globalement de mars à août en amont du lac, tandis que dans le lac même, il se reproduit de août à septembre, puis de février à juillet. Dans la rivière Agnébi, l'activité de reproduction est irrégulière. Nos observations sont semblables à celles de Albaret (1982). L'auteur indique que dans le fleuve Bandama (Côte d'Ivoire), l'activité reproductrice de $S$. schall semble atteindre un maximum en juin avec $90 \%$ des femelles en maturation avancée. Quant à Halim et Guma'a (1989), ils ont montré dans le Nil blanc près de Khartoum, (Soudan) que cette même espèce se reproduit de juillet à septembre. Willoughby (1979) l'observe en août dans le lac Kainji (Nigeria). Par ailleurs, selon Lowe Mcconnell (1987), Lam et Munro (1987) et Boujard (1992), dans les pays tropicaux, la pluviométrie exercerait une influence prépondérante sur le processus et la période de reproduction des poissons, à cause de l'alternance très marquée des saisons. $\mathrm{Ce}$ constat s'applique à nos résultats. En effet, la période de reproduction la plus importante qui part de mars à juin correspond en grande partie à la grande saison des pluies dans cette partie du pays. Ces résultats corroborent aussi ceux de Moreau et al., (1995), qui monrent que, la reproduction de $S$. schall survient lors de la montée des eaux en début des saisons de pluies. De façon globale, la permanence de l'eau dans le lac, expliquerait la plus longue période de reproduction observée en ce lieu par rapport aux parties fluviatiles des bassins étudiés, sujettes à de fortes périodes d'étiage. En outre, De Vlaming (1974) a indiqué que chez la plupart des animaux, l'activité de reproduction précède plus ou moins selon les caractéristiques du développement, une période où les facteurs du milieu (en particulier la nourriture disponible) sont les plus favorables à la survie des jeunes, donc à la pérennité de l'espèce. Dans le cas du poisson étudié, Diomandé (2001) a montré que dans le lac d'Ayamé I, au cours de la même période d'étude, le nombre d'aliments consommés par l'espèce est plus important en saison des pluies qu'en saison sèche, le ruissellement des eaux en direction du lac, mettant à sa disposition un plus grand nombre d'aliments. Cela se reflète en partie dans la structure des poids : les femelles du lac ont eu un poids corporel significativement plus élevé que celui des femelles de l'amont tel que montré plus haut. L'étude de la taille de première reproduction des femelles de $S$. schall, a montré également qu'elle est significativement plus grande dans le lac qu'en amont et dans la rivière Agnébi. Dans le bassin du Bandaman, Albaret (1982) a noté une taille de première maturité sexuelle comprise entre 150 et $155 \mathrm{~mm}$. Dans le lac Tchad, OforiDanson, (1992) a reporté pour cette même espèce, une taille de première reproduction de $200 \mathrm{~mm}$. Selon Albaret (1994), les variations rencontrées peuvent être imputables aux différentes stratégies développées dans des milieux différents pour une meilleure adaptation. Ces stratégies pouvant être d'ordre alimentaire ou de reproduction, ou les deux à la fois. Dans le cas présent, le grand nombre d'aliments présents dans le lac, comme indiqué plus haut, a favorisé la reproduction à une taille plus grande, tandis que le tarissement régulier, en amont, constituerait un facteur défavorable (stress) conduisant l'espèce à se reproduire à une plus petite taille.

Les paramètres biotiques (taille de première reproduction et poids corporel moyen) des femelles pêchées dans la rivière Agnébi ont été sensiblement voisins de ceux des femelles capturées dans le lac. En revanche, ils ont été significativement supérieurs aux mêmes paramètres des femelles récoltées en amont du lac, milieu également fluviatile comme l'Agnébi. Cette dernière situation montre que Synodontis schall non seulement se développe très bien en milieu lacustre, mais peut également le faire en zone fluviatile. Par conséquent, la présence des individus de petite taille et de faible poids en amont du lac a probablement été liée à une présence importante de juvéniles dans cette zone. En d'autres termes, une partie des populations de géniteurs migrerait du lac vers ses affluents en période de crue et se reproduirait en ces lieux. Ceci a déjà été observée par Hopson (1982), qui a indiqué que dans les grands lacs africains, $S$. schall migre dans des petits affluents temporaires pour se reproduire.

Relativement à la fécondité de l'espèce, l'analyse de variance montre, qu'il n'y a pas eu de différence significative concernant ce paramètre entre milieu lacustre et amont du lac. 
Ceci tend à confirmer l'hypothèse de migration pour la reproduction déjà évoquée.

La variation des différents paramètres de la reproduction en fonction des stations et des paramètres physico-chimiques montre que le Rapport Gonado-Somatique moyen (RGS moyen), le taux de maturation, de même que la longueur standard moyenne, le poids corporel moyen, caractéristiques de la croissance des individus, ont présenté les valeurs les plus élevées dans le lac. Le RGS et le taux de maturation sont, en particulier, fortement corrélés à la transparence et à la température du lac. La transparence élevée des eaux du lac, résultant de leur caractère relativement stagnant et du dépôt des matières en suspension qui l'accompagne, est un facteur qui favorise la photosynthèse algale.En conséquence, la productivité primaire sera augmentée et accompagnée d'une plus grande production d'oxygène disponible pour les poissons. Par ailleurs, la température élevée du lac s'explique par l'exposition permanente des eaux de surface au soleil (canopée nulle). Toutes ces considérations permettent d'affirmer que les nouvelles conditions environnementales apparues, suite à la mise en place du lac, favorisent la reproduction et la croissance de $S$. schall. Tout se passe comme si les paramètres environnementaux crééaient des meilleures conditions pour la survie des poissons (Légendre et Jalabert, 1988 ; Wootton, 1979).

\section{CONCLUSION}

Les paramètres de la reproduction et de la croissance de $S$. schall sont meilleurs en milieu lacustre qu'en milieu fluviatile. L'abondance d'aliments dans ce milieu et la température relativement élevée, seraient les facteurs favorisants. Par ailleurs, la reproduction de l'espèce a lieu en saison pluvieuse.

\section{REMERCIEMENTS}

Cette étude a été réalisée dans le cadre du Projet Ivoiro-belge Vlaamse Interuniversiteit Raad (VLIR/ KUL) intitulé «Evolution de la biodiversité après la construction d'un barrage : cas de la rivière Bia, Côte d'Ivoire», financé par l'Agence Générale Belge pour la Coopération au Développement. Nous voudrions à cet égard exprimer notre reconnaissance au Prof. Thys
Van den Audenaerde D. F. E. et à notre regretté Dr G. G. Teugels, respectivement promoteur et co-promoteur du Projet qui ont permis la réalisation de ce travail.

\section{REFERENCES}

Albaret (J. J.). 1982. Reproduction et fécondité des poissons d'eaux douces de Côte d'Ivoire. Rev. Hydrobiol. Trop. 15 (4) : 347371.

Albaret (J. J.). 1994. Les poissons, biologie et peuplements. In (J. R.) Durand ; (P.) Dufour ; (D.) Guiral et (S.G.F) Zabi (Ed.). Environnement et ressources aquatiques de Côte d'Ivoire. Tome II, ORSTOM, 238279.

Benech (V.) et (J.) Quensière. 1985. Stratégies de reproduction des poissons du Tchad en période de Tchad Normal (1966-1971). Rev. Hydrobiol. trop. 18 (3) : 227-244.

Boujard (T.). 1992. Space-time organization of revering fish communities in French Guiana. Env. Biol. Fish. 34 : 235-246.

De Vlaming (V. L.). 1974. Environmental and endocrine control of teleost reproduction. In (C.) Schreck (Ed.). Control of sex in Fishes. Sea Grant and V. P. I. and S. U. Press, 13-83.

Diomande (D.). 2001. Macro-faune benthique et stratégies alimentaires de Synodontis bastiani et $S$. schall en milieu fluvio-lacustre (Bassins Agnébi et Bia ; Côte d'Ivoire). Thèse de Doctorat. Université d'AboboAdjamé, $251 \mathrm{p}$.

Duponchelle (P.) and (M.) Legendre. 2000. Orechromis niloticus (Cichlidae) in lake Ayamé, Côte d'Ivoire : life history traits of a strong diminished population. Cybium 24 (2) : 161-172.

Durand (J. R.) et (G.) Loubens. 1970. Variations du coefficient de condition chez les Alestes baremoze du lac Tchad et du Bas Chari. Cahiers ORSTOM, série Hydrobiologie 4 (1) : 27-44.

Gourene (G.) ; (G. G.) Teugels et (D. F. E.) Thys Van Den Audenaerde. 1995. Manuel pratique d'identification des poissons du lac d'Ayamé (Rivière Bia, Côte d'Ivoire). Arch. Scient., CRO 14 (1) : 1- 41.

Halim (A. I. A.) and (S. A.) Guma'a. 1989. Some aspects of the reproductive biology of Synodontis schall (Bloch-Schneider, 1801) from the White Nile near Khartoum. Hydrobiologia 178 : 243-259. 
Hopson (A. J.). 1982. Lake Turkana. A report on the on the findings of the lake Turkana. Project 1972-1975. London Overseas Development Administration, 6, $1614 \mathrm{p}$.

Lam (T. J.) and (A. D.) Munro. 1987. Environmental control of reproduction in teleosts : an overview. Symposium on the reproductive physiology of fish, St John's, New-found land, 279-288.

Le Cren (E.D.). 1951. The length-weight relationship and seasonal cycle in gonad weight and condition in the perch (Perca fluviatilis). J. Anim. Ecol., 20 : 201-219.

Legendre (M.) et (B.) Jalabert. 1988. Physiologie de la reproduction. In (C.) Leveque ; (M.) Bruton et (G. W.) Ssentongo (Ed.). Biologie et écologie des poissons d'eau douce africains. Paris, ORSTOM, travaux et documents, 153-187.

Leveque (C.). 1999 a. Croissance et ontogénie in (C) Leveque et (D) Paugy (Ed.). Les poissons des eaux continentales africaines. Diversité, écologie, utilisation par l'homme, Paris ( IRD) : 153-166.

Leveque (C.). 1999 b. L'habitat des poissons. In (C) Leveque et (D) Paugy (Ed.). Les poissons des eaux continentales africaines. Diversité, écologie, utilisation par l'homme. Paris ( IRD) : 251-270.

Lowe-Mcconnell (R. H.). 1987. Ecological studies in tropical fish communities. Cambridge, University Press, Tropical Biology series, $382 \mathrm{p}$.

Moreau (J.). 1979. Biologie et évolution des peuplements de Cichlidae introduits dans les lacs malgaches d'altitude. Thèse de Doctorat d'Etat, INP, Toulouse, 345 p.

Moreau (J.) ; (M. L. D.) Palomares, (F. S. B.) Torres J. R. et (D.) Pauly. 1995. Atlas démographique des populations de poissons d'eau douce d'Afrique. ICLARM, Rapp. Tech. 45, 140 p.

Munro (A. D.). 1990. Tropical freshwater fish. In (A. D.) Munro ; (A. P.) Scott and (T. J.) Lam (Ed.). Reproduction and seasonality in teleosts : environmental influences Boca Raton, Florida, CRC Press, 145- 239.

Ofori-Danson (P. K). 1992. Ecology of some species of catfish Synodontis (Pisces : Mochokidae) in the Kpong Headpond in Ghana. Env. Biol. Fish 35 : 49-61.

Omanney (F. D.). 1964. Les complexités de la reproduction. In les poissons. Collection LIFE, $192 p$.

Paugy (D.) et (T. R.) Roberts. 1992. Mochokidae. In (C.) Lévêque, (D.) Paugy et (G. G.) Teugels, (Ed.). Faune des poissons d'eaux douces et saumâtres de l'Afrique de l'Ouest. Tome II, ORSTOM / MRAC, 500-563.

Vanden Bossche (J. P.) and (G. M.) Bernacsek. 1990. Source book for the inland fishery resources of Africa. FAO, 2. 240 p.

Willoughby (N. G.). 1979. Some aspects of the ecology of Synodontis (pisces : siluroidae) in Lake Kainji during its early years. Presented to the international Conference on Kainji Lake and River Basins development in Africa. Ibadan, 376- 392.

Wootton (R. J.). 1979. Energy cost of eggs production and environmental determinants of fecundity in teleost fishes. In (P. J.) Miller (Ed.). Fish phenology : anabolic adaptativeness in teleosts. Symp. zool. Soc. London, 44, 133-159.

Wootton (R. J.). 1990. Ecology of Teleost fishes. London, Chapman and Hall, Fish and Fisheries series, $21: 404 \mathrm{p}$. 